\title{
Tuning Liposome Membrane Permeability by Competitive Coiled Coil Heterodimerization and Heterodimer Exchange
}

Camilla Skyttner, Karin Enander, Christopher Aronsson and Daniel Aili

The self-archived postprint version of this journal article is available at Linköping University Institutional Repository (DiVA):

http:/ / urn.kb.se/ resolve?urn=urn:nbn:se:liu:diva- 149349

N.B.: When citing this work, cite the original publication.

Skyttner, C., Enander, K., Aronsson, C., Aili, D., (2018), Tuning Liposome Membrane Permeability by Competitive Coiled Coil Heterodimerization and Heterodimer Exchange, Langmuir, 34(22), 65296537. https:// doi.org/ 10.1021/ acs.langmuir.8b00592

Original publication available at:

https:/ / doi.org/ 10.1021/ acs.langmuir.8b00592

Copyright: American Chemical Society

http:// pubs.acs.org/ 


\title{
Tuning Liposome Membrane Permeability by
}

\section{Competitive Coiled Coil Heterodimerization and}

\section{Heterodimer Exchange}

Camilla Skyttner, Karin Enander, Christopher Aronsson, Daniel Aili*

Division of Molecular Physics, Department of Physics, Chemistry and Biology, Linköping University, 58183 Linköping, Sweden

KEYWORDS Liposome, Membrane Active Peptide, Coiled Coil, Heterodimer, Drug Delivery System

\begin{abstract}
Membrane active peptides that enable triggered release of liposomal cargo is of large interest for development of liposome-based drug delivery systems but require peptide-lipid membrane interactions that are highly defined and tunable. To this end, we have explored the possibility to use the competing interactions between membrane partitioning and heterodimerization and folding of a set of four different de novo designed coiled coil peptides. Covalent conjugation of the cationic
\end{abstract}


peptides triggered rapid destabilization of membrane integrity and release of encapsulated species. The release was inhibited when introducing complementary peptides as a result of heterodimerization and folding into coiled coils. The degree of inhibition was shown to be dictated by the coiled coil peptide heterodimer dissociation constants, and liposomal release could be reactivated by a heterodimer exchange to render the membrane bound peptide free and thus membrane active. The possibility to tune the permeability of lipid membranes using highly specific peptide folding dependent interactions delineates a new possible approach for further development of responsive liposome-based drug delivery systems.

\section{INTRODUCTION}

Membrane active peptides are an important class of functional biomolecules that are found in virtually all living organisms and able to act as cell-penetrating agents (CPPs) $)^{1}$ to facilitate cell membrane translocation, or provide antimicrobial, antiviral, and antifungal activity. ${ }^{2-4}$ The variations in amino acid composition, structure, and function of membrane active peptides are very large but cationic and amphipathic peptides are overrepresented, especially among antimicrobial peptides (AMPs). AMPs tend to kill bacteria by compromising the integrity of the lipid cell membrane in a pore-formation process or a as result of a detergent-like carpet mechanism. Membrane active peptides have been extensively investigated for various therapeutic applications, including CPP-based drug delivery systems ${ }^{5,6}$ and as AMP-derived anti-infective drugs. ${ }^{7}$ Both natural peptides, derivatives of natural peptides and fully synthetic and designed membrane active peptides have been explored. ${ }^{8}$ To increase lipid membrane affinity of peptides with no or very 
little membrane activity, lipidation of peptides with a hydrophobic moiety, such as phospholipids, cholesterol or fatty acids, has also been extensively investigated. ${ }^{9-11}$

The ability of membrane active peptides to increase the permeability of lipid membranes has also been investigated as a means to release liposome encapsulated cargo in drug delivery applications. ${ }^{12-14}$ Liposomes are widely used in drug delivery and low molecular weight drugs can often be efficiently encapsulated in either the aqueous inner compartment or in the lipid bilayer. ${ }^{15}$ When combined with techniques for obtaining stable liposomes with long circulation times, significant improvements in drug pharmacokinetics can be achieved. ${ }^{16-18}$ Presently there are about ten liposome-based drug formulations in clinical use for treatment of e.g. breast cancer, ovarian cancer, and leukaemia, and a large number of liposome-based drug formulations are in various stages of clinical trials. ${ }^{2,19}$ Peptide-functionalization of liposomes is an important strategy in the design of drug delivery systems that recognize cell surface receptors or respond to specific enzymes, such as proteases, which can enable more efficient and specific release of encapsulated drugs or stimulate endocytic uptake of the liposomes. ${ }^{14,20-22}$ However, design of drug delivery systems exploiting peptide-mediated interactions for triggered release require well-defined peptide-lipid interactions that can be rationally tailored and controlled. We have previously demonstrated a de novo designed amphipathic helix-loop-helix peptide that is membrane active only when covalently bound to a lipid membrane. ${ }^{13}$ Furthermore, the release of liposomal cargo was inhibited upon dimerization with a complementary peptide. The inhibitory effect was downregulated by specific proteolytic digestion of the complementary peptide by matrix metalloproteinase 7 (MMP-7).

Here we show a set of de novo designed coiled coil peptides ${ }^{23,24}$ that constitute a similar but more sophisticated toolbox for tailoring the permeability of liposomes. The 28 residue peptides EI, EV, 
KI and KV, with sequences based on peptides designed by Woolfson et al. ${ }^{25}$ and Hodges et al., ${ }^{26,27}$ are random coils as monomers at room temperature but can heterodimerize and fold into coiled coils. Four different parallel heterodimers (EIKI, EIKV, EVKI and EVKV) can be obtained showing affinities for dimerization ranging from the micromolar to the picomolar range. The peptides are amphipathic and the lysine-rich $\mathrm{KI}$ and $\mathrm{KV}$ have a positive net charge at neutral $\mathrm{pH}$ whereas the glutamic acid-rich EI and EV have a negative net charge at neutral $\mathrm{pH}$. To enable covalent conjugation of the cationic peptides to lipids in liposomes, KI and KV were modified with an N-terminal Cys, yielding the peptides KIC and KVC, respectively. The cationic peptides KIC and KVC were membrane active only when covalently bound to a lipid membrane and anchoring to liposomes lead to rapid and peptide concentration dependent release of encapsulated fluorophores. KIC and KVC displayed a significant difference in release rate and extent despite minor differences in amino acid composition. Heterodimerization with the complementary peptides, EI and EV, inhibited release of liposomal content. Removal of the complementary peptide by a competitive heterodimer exchange reactivated the membrane active peptide thus enabling a triggered release of liposomal content. The possibility to utilize highly specific peptide folding dependent interactions to tune the permeability of lipid membranes offers both an insight into the complexity of peptide-lipid membrane interactions and an interesting approach for further development of responsive liposome-based drug delivery systems.

\section{RESULTS AND DISCUSSION}

Peptide anchoring to liposomes and liposome cargo release. The peptides EI, EV, KI, and KV are amphipathic and designed to heterodimerize and fold into coiled coils (Figure S1). ${ }^{23}$ The main 
driving force for folding is the burial of hydrophobic residues (Val and Ile) in the hydrophobic core. In addition, interactions between charged residues (Glu and Lys) located at the dimer interface promote formation of heterodimers over homodimers. KI and KV were further modified with a Cys in the N-terminus to generate the peptides KIC and KVC. The Cys residue enables covalent coupling of the peptides to lipids functionalized with a maleimide headgroup (MPB-PE) by means of a thiol-maleimide Michael addition. ${ }^{24}$ In order to investigate the effects of membrane anchoring of KIC and KVC on liposome membrane integrity, 5(6)-carboxyfluorescein (CF) was encapsulated at self-quenching concentration (50 mM) in POPC liposomes with 0,1 , and 5 mol\% MPB-PE (Figure S2), corresponding to a MPB-PE concentration of $0,0.4$, and $2 \mu \mathrm{M}$, respectively. The thiol moiety in the N-terminal Cys residue of KIC and KVC can primarily react with the MPB$\mathrm{PE}$ in the outer lipid leaflet resulting in a covalent conjugation of the peptides to lipids in the liposome bilayer. Membrane permeability was exclusively increased when KIC or KVC were added to liposomes with 5 mol\% MPB-PE, observed as a rapid and peptide concentration dependent release of encapsulated CF (Figure 1A-C and Figure S3). The liposomes alone released less than 3\% CF during $2 \mathrm{~h}$ (Figure S4). In the absence of MPB-PE (0 mol\%), no increase in CF release was detected upon addition of KIC or KVC, nor was any release observed at 5 mol\% MPBPE when the Cys residue in the peptides was excluded (Figure S5A-B). Covalent anchoring of the peptides to MPB-PE in the liposomes was thus consequently a prerequisite for the observed CF release. In addition, oxidation of the Cys residue, which results in disulfide bond formation between two peptides, precludes the binding of the peptides to MPB-PE and resulted in a drastic reduction in the release (Figure S6). The experimental conditions were thus chosen to ensure that the majority of the peptides (more than $85 \%$ ) would remain in their reduced form for the duration of the experiments. The rate of oxidation, determined using an Ellman's test for free thiols, ${ }^{28}$ 
differed significantly for KIC and KVC. KVC remained in the reduced form for more than $8 \mathrm{~h}$ whereas KIC oxidized faster and already after $1.5 \mathrm{~h}$ the oxidation was about $20 \%$ when incubated at $100 \mu \mathrm{M}$ in PBS at room temperature (RT) (Figure S7). This difference in oxidation rate is likely a consequence of the higher propensity for homodimerization of $\mathrm{KI}$ in PBS as compared to KV, as previously described by Aronsson et al. ${ }^{23}$ KIC has isoleucine (Ile) in position $a$ and alanine (Ala) in position $c$ in the heptad repeat whereas KVC has valine (Val) and serine (Ser) in these positions (Figure S1). Due to the higher packing efficiency of Ile as compared to Val in the hydrophobic core along with the higher $\alpha$-helical propensity of Ala compared to Ser, ${ }^{26} \mathrm{KIC}$ is more prone to homodimerization than KVC. Homodimerization of KIC and KVC leads to a closer alignment of the Cys residues in the two peptides, which facilitates intermolecular disulfide formation.

A

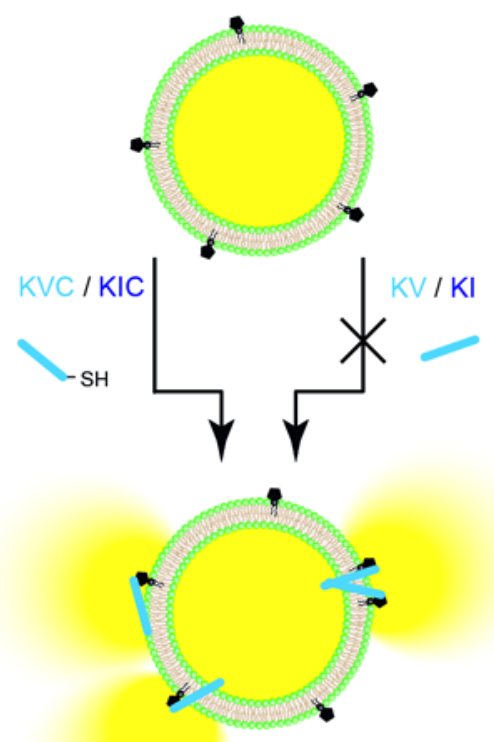

B

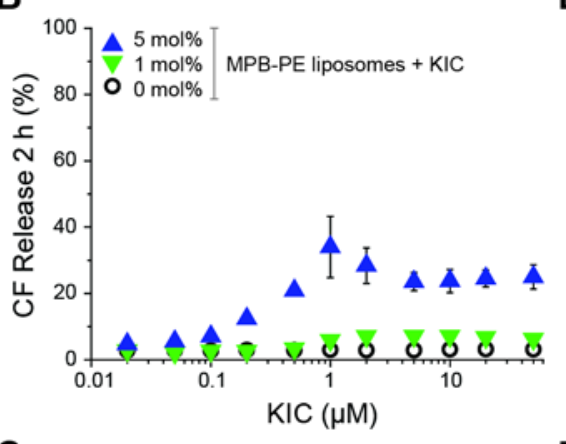

C

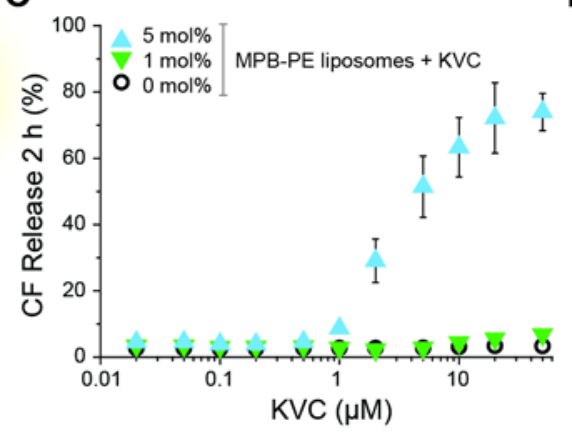

D

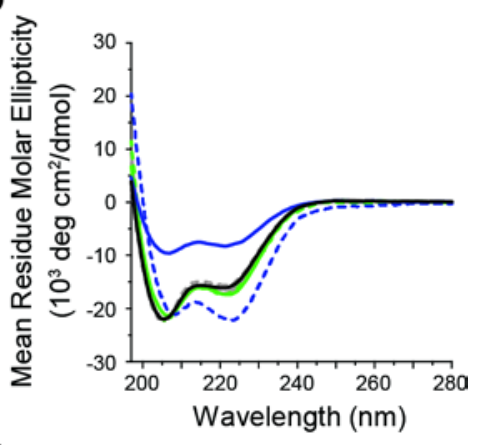

E

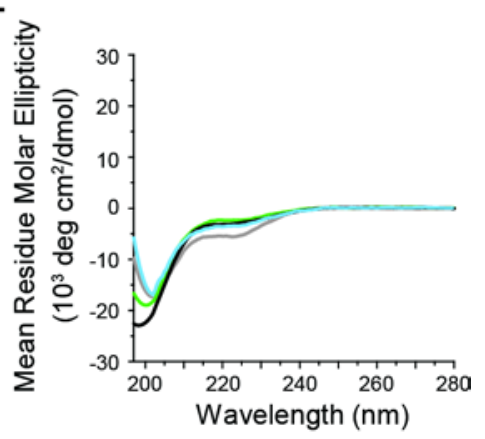


Figure 1: A) Schematic illustration representing the binding of KIC/KVC and the non-binding of $\mathrm{KI} / \mathrm{KV}$ to the MPB-PE-containing liposomes. Release of CF after $2 \mathrm{~h}$ upon addition of 0.01-50 $\mu \mathrm{M} \mathrm{B}$ ) KIC and C) KVC to liposomes containing 5 mol\% (dark and light blue triangle, respectively), 1 mol\% (green triangle) and 0 mol\% (black circle) MPB-PE in a total lipid concentration of $40 \mu \mathrm{M}$ in PBS with a peptide:maleimide ratio ranging from 0:0-25:1. CF release without peptide addition was $<3 \%$ for all liposome compositions. The CF release was measured every 2 min for 2 h, Figure S3. CD spectra of $50 \mu \mathrm{M}$ D) KIC and E) KVC without (black) and with liposomes incubated for 10 min (dashed line, only in D) and 8 h (solid line) containing 0 mol\% (grey) 1 mol\% (green) and 5 mol\% MPB-PE (dark and light blue, respectively) with a total lipid concentration of $1 \mathrm{mM}$ in PBS with a peptide:maleimide ratio of 50:0, 5:1 and 1:1, with respect to liposome composition.

The minimum concentration needed for significant CF release (defined as twice the maximum CF release from the liposomes alone, i.e. $>6 \%$, for 5 mol\% MPB-PE liposomes, Figure S4) was 0.1 $\mu \mathrm{M}$ for KIC and $1 \mu \mathrm{M}$ for $\mathrm{KVC}$ when added to liposomes with $5 \mathrm{~mol} \% \mathrm{MPB}-\mathrm{PE}$. The release efficiency increased for KIC concentrations up to $1 \mu \mathrm{M}$. However, at higher peptide concentrations the release decreased slightly to a level between 20 and 30\% (Figure 1B). This indicates that KIC reached a threshold concentration $(>1 \mu \mathrm{M})$ where the interaction with the lipid membrane appeared to be less favorable than homodimerization. The release caused by addition of KVC, on the other hand, increased with increasing concentrations of the peptide up to about $10 \mu \mathrm{M}$ (Figure 1C). In addition to the different preferences for homodimerization, the markedly different CF release profiles for the two peptides is also likely a consequence of the small differences in hydrophobicity. The hydropathy index, as described by Kyte and Doolittle ${ }^{29}$, is higher for Ile (4.5) than for Val 
(4.2). Also, the free energy of transfer from lipid bilayer to water measured by Wimley and White ${ }^{30}$ proved Ile to have a more favorable environment in the lipid bilayer compared to Val $\left(\Delta \mathrm{G}_{\text {residue }}=\right.$ $0.3 \mathrm{kcal} / \mathrm{mol}$ and $-0.1 \mathrm{kcal} / \mathrm{mol}$ respectively) when the residues resided in a short peptide.

Interestingly, the CF release for the liposomes with 1 mol\% MPB-PE was very low, even at high concentrations of KIC or KVC. If saturated, the surface concentration of peptides on liposomes with 1 mol\% MPB-PE will be more than twice than the theoretical maximum for 5 mol\% MPBPE exposed to $0.2 \mu \mathrm{M}$ KIC. Yet, the latter produced a significantly higher release than what was observed for liposomes with 1 mol\% MPB-PE at any peptide concentration, which indicates that the number of possible anchoring points are just as important as the final peptide surface concentration.

Peptide-liposome interactions. The effects of liposome anchoring and peptide oxidation on the secondary structure was investigated using circular dichroism spectroscopy (CD). The CD spectra were acquired using a peptide:maleimide ratio of 1:1 after 8 hours incubation to ensure the contribution of the unconjugated peptides would be negligible. Reduced and unbound KIC in PBS buffer showed an $\alpha$-helical content that increased when anchored to 5 mol\% MPB-PE (Figure 1D). In the absence of liposomes (Figure S8), the $\alpha$-helical content of KIC also steadily increased, likely due to oxidation of the Cys which further facilitated homodimerization. In the absence of liposomes the process was, however, much slower and required about 3 days of incubation in PBS to show similar helicity as when incubated for 10 minutes with liposomes with 5 mol\% MPB-PE. The secondary structure of free reduced and liposome anchored KIC showed interesting differences, clearly seen when examining the ratio of the characteristic minima at 208 and $222 \mathrm{~nm}$ 
in the CD spectra $\left(\Theta_{222 / 208}\right)$. Reduced and unbound KIC showed $\Theta_{222 / 208}=0.81$, that increased slightly to $\Theta_{222 / 208}=0.83$ after $1 \mathrm{~h}$ incubation in PBS. This ratio was significantly higher for KIC with 5 mol\% MPB-PE liposomes $\left(\Theta_{222 / 208}=1.05\right)$ already after 10 min incubation. Typically, well defined coiled coils display $\Theta_{222 / 208}>1,{ }^{31}$ suggesting that KIC adopted a defined coiled coil structure when anchored to the liposome membrane. This is likely a consequence of peptides associating and homodimerizing either laterally in the lipid bilayer or by interactions between peptides anchored on separate liposomes. Interliposomal interactions promoted by peptide homodimerization were indicated by a drastic drop in the CD signal intensity over time, likely due to aggregation of the liposomes. Aggregation was confirmed by dynamic light scattering (DLS) in samples containing KIC and 5 mol\% MPB-PE liposomes (Figure 2). The aggregation process was rapid and an increase in hydrodynamic radius was observed within minutes after KIC was added to the MPB-PE liposomes. The aggregate size increased over a period of $4 \mathrm{~h}$ resulting in macroscopic precipitates visible by eye. The change in secondary structure of KIC was likely a contributing factor to the liposome aggregation, and it cannot be determined if it also was a part of the liposome release mechanism. 


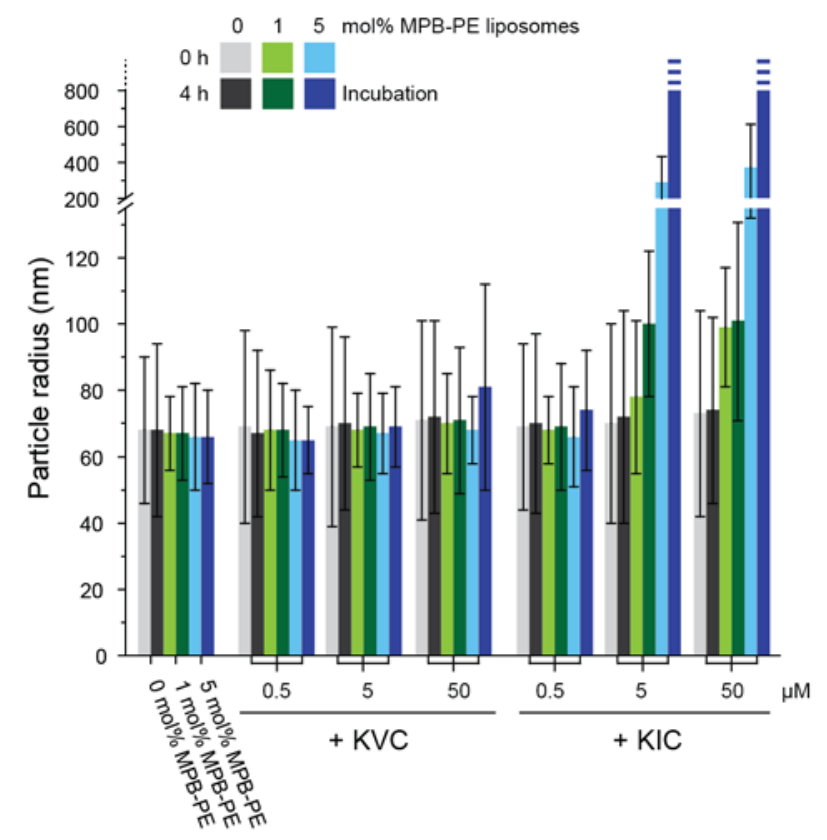

Figure 2: Hydrodynamic radius of liposomes with 0 (light and dark grey), 1 (light and dark green) and 5 (light and dark blue) mol\% MPB-PE after 0 h (light colors) and 4 h (dark colors) incubation with $0.5,5$ and $50 \mu \mathrm{M}$ KVC or KIC and a total lipid concentration of $40 \mu \mathrm{M}$ and a peptide:maleimide ratio of 1:4, 5:2, 25:1 (when using 5 mol\% MPB-PE liposomes).

Interestingly, while being efficient in releasing encapsulated CF, KVC did not show any defined secondary structure when anchored to MPB-PE liposomes (Figure 1E) despite being designed to adopt a coiled coil motif similar to $\mathrm{KIC} .^{23,24}$ Compared to KIC, KVC was less prone to homodimerize due to the substitution of Ile for Val in the hydrophobic core (Figure S1), ${ }^{23}$ and was thus more likely to partition into the lipid membrane to shield its hydrophobic residues which induced a change in the liposome permeability. Commonly, partitioning of amphipathic peptides into lipid membranes is accompanied by formation of secondary structure, so called partitionfolding coupling. ${ }^{32}$ This raises the question whether KVC resides on the surface or inside the lipid 
bilayer or if only a small fraction of the peptides was inserted into the lipid bilayer making assessment of the change in secondary using CD spectroscopy structure difficult.

In contrast to KIC, no major changes in the hydrodynamic radius of liposomes with and without MPB-PE were seen upon addition of KVC (Figure 2). Addition of KVC to MPB-PE liposomes resulted in a slight increase $(1-15 \mathrm{~nm})$ in radius, likely as a result of the anchoring of the peptides to the liposomes. DLS did not show any smaller fragments (e.g. micelles) even at the highest peptide:maleimide ratio of 25:1 (50 $\mu \mathrm{M}$ KVC +5 mol\% MPB-PE, Figure S9) nor a decrease in count rate (241 kcps and 245 kcps before and after peptide addition, respectively), indicating that the liposomes remained intact albeit the pronounced loss in membrane integrity as shown by the rapid CF release. These findings were further supported by surface plasmon resonance (SPR) data on liposomes tethered to a Biacore L1 chip (Figure 3). The injection of KVC over a surface with 5 mol\% MPB-PE liposomes resulted in a response of 700-1200 RU (10 min contact time) corresponding to a surface concentration of peptides that correlated well with the amount of available maleimides on the MPB-PE liposomes (Figure 3B). Since no loss of material could be observed during or after the anchoring of the peptides, a peptide-lipid interaction via a detergentlike carpet mechanism ${ }^{33}$ seems unlikely. The injection of KIC over a surface with immobilized 5 mol\% MPB-PE liposomes resulted in a higher degree of conjugation than expected considering the amount of available maleimides. This further indicates that homodimerization between conjugated and unconjugated KIC can occur, resulting in association and recruitment of additional peptides to the liposomes surface. This effect was also previously observed for a related designed cationic amphipathic helix-loop-helix peptide studied under similar conditions, but did not inhibit the release as was seen here. ${ }^{13}$ 

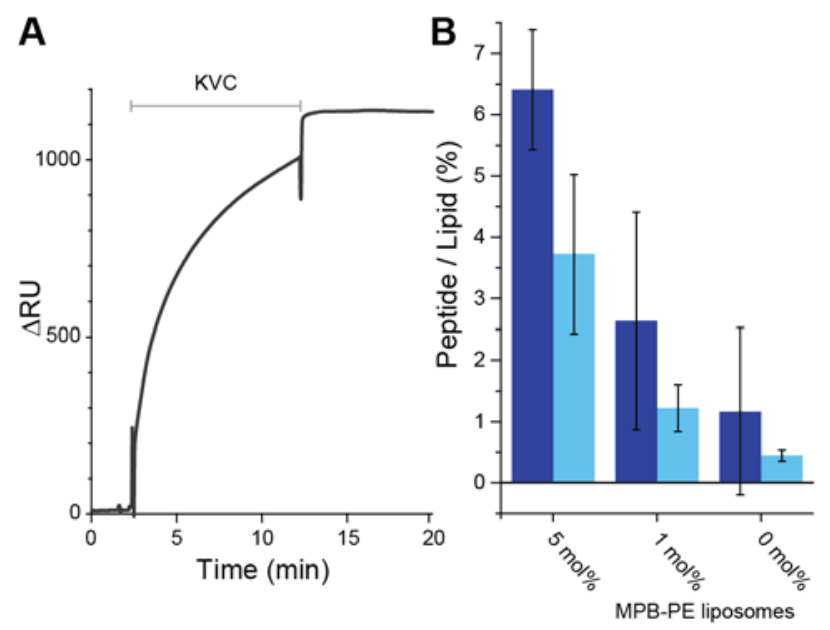

Figure 3: SPR measurements of peptides interacting with liposomes immobilized on a L1 sensor chip. A) Representative SPR sensogram showing the immobilization of KVC on a L1 sensor chip with immobilized 5 mol\% MPB-PE liposomes. B) The amount of peptide per lipid on liposomes with 0, 1 and 5 mol\% MPB-PE after a 10 min KIC (dark blue) KVC (light blue) injection (200 $\mu \mathrm{M})$. Reference injections with $\mathrm{KI} / \mathrm{KV}$ showed very limited binding to the sensor surfaces and this data was subtracted from the data to account for buffer and background effects.

In order to further elucidate the nature of the interaction between KVC and the lipid membrane, isothermal titration calorimetry (ITC) was carried out. The interpretation of the ITC data was complicated by the fact that both covalent and non-covalent interactions contribute. Addition of Cys alone to liposomes with 5 mol\% MPB-PE showed a highly exothermic $(\Delta \mathrm{H}=-0.57 \mathrm{kcal} / \mathrm{mol})$ reaction as expected (Figure S10). The final molar ratio of Cys to maleimides was roughly 4:5, which indicates that the majority of maleimides in the MPB-PE liposomes were available for binding, likely due to transbilayer movement of mainly unconjugated and perhaps Cys conjugated MPB-PE. ${ }^{34}$ Addition of KVC to liposomes with 0 mol\% MPB-PE indicated, as expected, no obvious interactions. Liposomes with 5 mol\% MPB-PE, on the other hand, showed roughly a 1:2 
KVC:MPB-PE stoichiometry and an exothermic reaction $(\Delta \mathrm{H}=-0.22 \mathrm{kcal} / \mathrm{mol})$ with contributions from both the Cys mediated peptide conjugation and physical peptide-lipid membrane interactions. The lower enthalpy for binding of the peptide to the liposomes compared with Cys alone, indicated that the peptide-lipid interaction was endothermic. The interaction with the lipid membrane was hence driven by a combination of a proximity effect caused by the formation of the covalent thiolmaleimide bond and a potential entropic gain upon membrane partitioning that is not fully compensated for in Gibbs free energy of folding, resulting in a less pronounced partition-folding coupling as indicated by the CD spectra. Still, the association of the peptide with the lipid membrane resulted in a definite destabilization in lipid membrane integrity which resulted in liposome release above a threshold concentration of anchored peptides.

Inhibition of liposome cargo release by peptide heterodimerization. To further investigate the possibility to utilize specific peptide-mediated interactions to tune the release of CF, the peptides EI and EV were used to introduce a competitive interaction. These experiments were done with KVC only since KIC showed such strong tendencies for homodimerization. Upon heterodimerization and folding of the peptides into coiled coils, the Cys residue in KVC will still be accessible for binding to the MPB-PE liposomes although the hydrophobic residues will no longer be available for interactions with the lipid bilayer. The release of encapsulated CF by KVC was consequently inhibited when KVC was first allowed to heterodimerize with the charge complementary peptides EI or EV prior to anchoring to liposomes (Figure 4). As the dimeric coiled coils are in equilibrium with their monomeric constituents a fraction of the peptides will, however, be capable of interacting with the lipid bilayer. The inhibitory effect was thus dependent on the affinity for heterodimerization. The higher the dissociation constant the more of the anchored KVC 
existed as monomers (Figure S11) that could interact with the lipid membrane, causing release. The inhibition was thus always more effective when using EI as compared to EV at the same concentrations (Figure 4B-C). The CF release caused by $5 \mu \mathrm{M} \mathrm{KVC}$ was $45 \%$ in the absence of inhibitory peptides. The addition of EV (50 $\mu \mathrm{M})$ reduced the CF release to $26 \%$ while EI (50 $\mu \mathrm{M})$ reduced the release to $11 \%$ after $4 \mathrm{~h}$ exposure to $5 \mathrm{~mol} \%$ MPB-PE liposomes. Similar inhibitory results were obtained for $10 \mu \mathrm{M} \mathrm{KVC}$ where the release was $58 \%$ in the absence of EI and EV, which was reduced to $25 \%$ and $46 \%$ in the presence of EI and EV, respectively. The CF release caused by EI and EV alone was negligible (less than 5 \%) for all liposome compositions and peptide concentrations (Figure S5C-D) strongly indicating that the anionic peptides did not interact with the lipid membrane. 

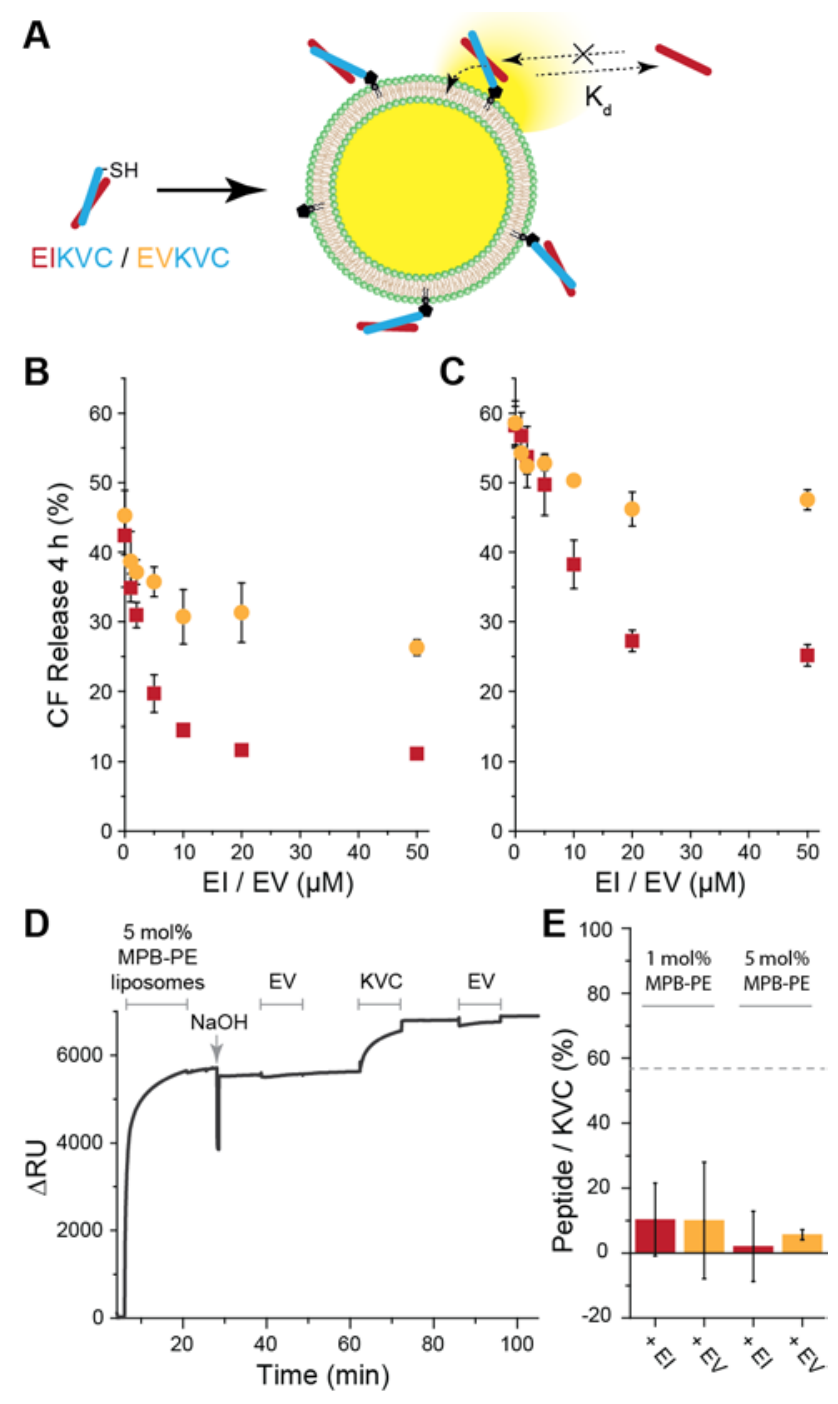

Figure 4: A) Allowing KVC to heterodimerize and fold into coiled coils with any of the complementary peptides EI or EV led to a significant reduction in CF release. CF release of 5 mol\% MPB-PE liposomes functionalized with heterodimers of EVKVC (orange circles) or EIKVC (red squares) measured after $4 \mathrm{~h}$ using B) $5 \mu \mathrm{M}$ and C) $10 \mu \mathrm{M} \mathrm{KVC}$ and varying concentrations of the complementary peptides EI and EV and using a total lipid concentration of $40 \mu \mathrm{M}$ and a peptide(KVC):maleimide ratio of 5:2 and 5:1 in B and C, respectively. D) Representative SPR sensorgram showing immobilization of liposomes with 5 mol\% MPB-PE, rinsing with $\mathrm{NaOH}$, and subsequent peptide injections $(200 \mu \mathrm{M})$ as indicated. E) The amount of EI or EV bound to KVC- 
functionalized liposomes after a 10 min injection of $200 \mu \mathrm{M}$ the peptides. Injections of the same concentration of EL/EV over unfunctionalized liposomes with the same lipid composition were used as reference. The grey dashed line indicates the corresponding maximum level of EIKVC on a Biacore CM5 sensor chip, where KVC was immobilized to the sensor chip via thiol coupling prior injection of EI ( $200 \mu \mathrm{M})$.

Moreover, when KVC was anchored to MPB-PE liposomes, a subsequent injection of any of the complementary peptides EI or EV, resulted in very limited interactions between KVC and EI/EV (Figure 4D-E, and Figure S12). In contrast, when KVC was instead immobilized on a sensor chip with a carboxymethylated dextran matrix (Biacore CM5) and exposed to EI/EV, about $60 \%$ of the immobilized KVC peptides were in the heterodimeric state (Figure 4E). Liposome anchored KVC was hence prevented from heterodimerizing with EI or EV despite the relatively high affinities for dimerization $\left(\mathrm{K}_{\mathrm{d}}(\mathrm{EIKV})=0.072 \mu \mathrm{M} \text { and } \mathrm{K}_{\mathrm{d}}(\mathrm{EVKV})=1.4 \mu \mathrm{M}\right)^{23}$. Note that this was also true for KVC attached to 1 mol\% MPB-PE liposomes (Figure 4E), even though no increased membrane permeability was observed under these conditions (Figure 1C). This indicates that all bound KVC peptides have relatively strong interaction with lipids in the membrane restricting access to interactions with the complementary peptides.

Heterodimer exchange for triggered release. The association and folding of the coiled coils is a dynamic process and the competitive interactions of dimerization and membrane partition, where the latter appears to be energetically more favorable, will eventually lead to dissociation of the heterodimers. This process is slow and dependent on the dissociation rate of the heterodimeric 
complex. However, introducing a second competing interaction can enable modulation of the dissociation rate of the membrane bound heterodimers. The peptides used in this work were designed to be promiscuous, meaning that no negative design elements were introduced to prevent certain heterodimers from forming. Thus, based on the four peptides, four different heterodimers can be obtained but with significant differences in affinities for dimerization. The differences in affinities result in social self-sorting of heterodimers in solution, ${ }^{23}$ but could also be utilized to dynamically dissociate the inhibiting EI peptide when associated to the liposome anchored KVC. Additions of KI or KV to anchored heterodimers of EIKVC hence resulted in reactivation of the CF release in a concentration dependent manner (Figure 5). Addition of KV/KI at the same, or higher, concentrations as the inhibiting peptide (EI) almost reestablished the release to the same level as in the control liposomes, i.e. without the addition of EI. At the time scales used here, i.e. 4 hours of incubation, both KV and KI showed about the same capacity to reactivate the release. In addition, the tendency of KI to homodimerize at RT (Figure S7, Figure S8 and Aronsson et. al. ${ }^{23}$ ) could also slightly reduced its efficiency in promoting the heterodimer exchange. 


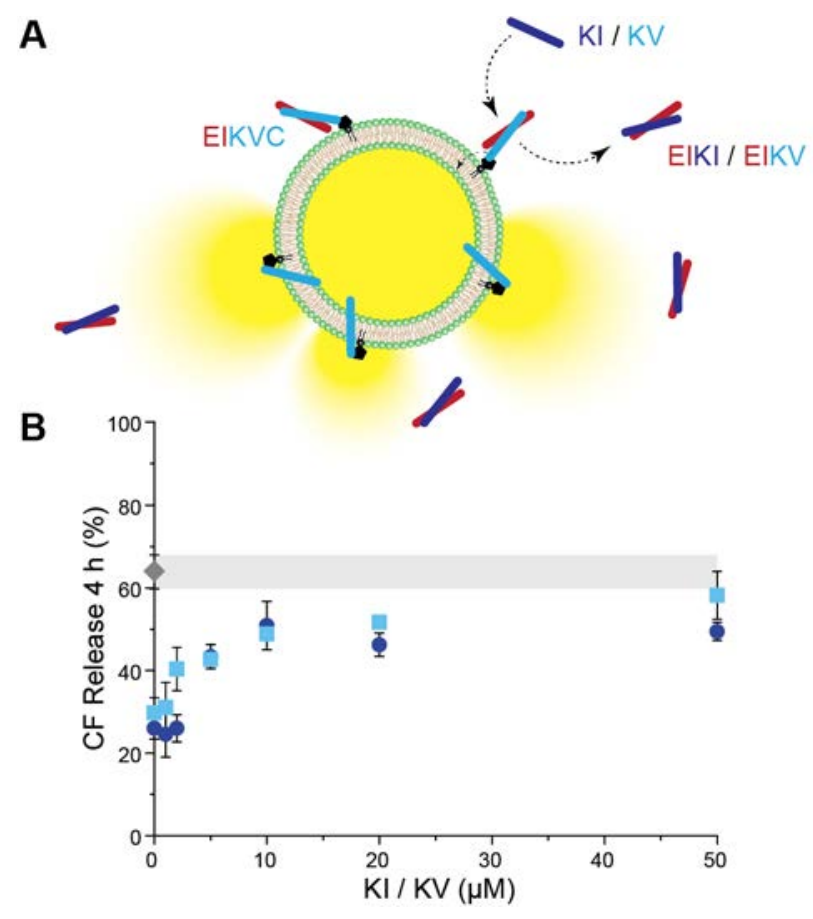

Figure 5: A) Schematic illustration of the heterodimer exchange upon addition of KI or KV leading to dissociation of the membrane bound heterodimer EIKVC and reactivation of the membrane destabilizing effect of KVC. B) $5 \mu \mathrm{M} \mathrm{KVC}+10 \mu \mathrm{M} \mathrm{EI}+\mathrm{KI}$ (dark blue) or KV (light blue) and the control $5 \mu \mathrm{M} \mathrm{KVC} \mathrm{(grey).} \mathrm{All} \mathrm{additions} \mathrm{of} \mathrm{KI} \mathrm{and} \mathrm{KV} \mathrm{were} \mathrm{done} \mathrm{with} \mathrm{the} \mathrm{same}$ added volume to avoid differences in dilution effects and the total lipid concentration was $40 \mu \mathrm{M}$ and a peptide(KVC):maleimide ratio of 5:2.

The accelerated release upon addition of KV/KI is likely preceded by formation of an intermediate peptide multimer, which was clearly indicated when investigating the interaction using SPR (Figure 6). Since KVC will not be accessible for heterodimerization after immobilization on liposomes, the heterodimer exchange was investigated using a sensor chip with a carboxymethylated dextran matrix (Biacore CM5). KVC was immobilized on the CM5 chip using thiol coupling. Subsequent injection of EI resulted in formation of heterodimers that, under the 
flow conditions used here, started to dissociate immediately after the injection had finished. After an initially rapid dissociation, leaving about $200 \mathrm{RU}$ of the heterodimers on the surface, a second injection of $\mathrm{KI}$ or $\mathrm{KV}$ resulted in a distinct association of peptides. The responses were comparable for both peptides and inversely proportional to the concentration of $\mathrm{KV} / \mathrm{KI}$. The higher the concentration of KV/KI the faster the dissociation of the multimeric complex. Neither KV nor KI showed any association to immobilized KVC nor to a Cys deactivated reference channel (data not shown). The association of intermediate multimers during the heterodimer exchange process was also seen when the anionic peptides were immobilized on the CM5 chips (Figure S13).
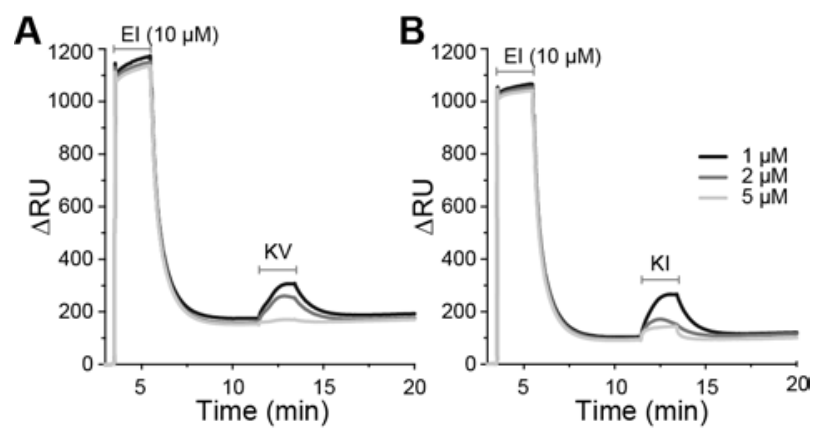

Figure 6: EI $(10 \mu \mathrm{M})$ introduced to a Biacore CM5 chip with immobilized with KVC followed by a subsequent addition of 1, 2 and $5 \mu \mathrm{M} \mathrm{A}$ ) KV and B) KI. Heterodimers are present on the sensor chip after injection of EI but to a low extent. The subtracted reference with only Cys on the surface showed no binding of either EI, KI or KV (not shown).

CONCLUSION 
Tailoring of liposome permeability is of large interest in drug delivery applications to ensure optimized release profiles. To this end, we have investigated the possibility to utilize a set of de novo designed coiled coil polypeptides for tuning liposomal integrity. The two cationic amphipathic peptides KIC and KVC were both found to trigger the release of liposome encapsulated carboxyfluorescein (CF), but only when covalently anchored to the lipid membranes. The relatively small difference in sequence between KIC and KVC (Figure S1) resulted in large differences in CF release, where KIC was more effective than KVC at low concentrations $(0.1 \mu \mathrm{M}$ $<\mathrm{KIC} / \mathrm{KVC}<5 \mu \mathrm{M})$. At higher concentrations, KIC showed tendencies for homodimerization which impaired the release and lead to liposome aggregation. KVC, on the other hand, triggered an almost complete CF release with only minor effects on liposomal size. Interestingly, despite clearly associating with the membrane KVC remained as random coil, which is in contrast to other lipidated peptides with similar primary structures. ${ }^{13,35}$ Isothermal calorimetry revealed that the peptide-lipid interaction was endothermic and thus likely driven by proximity effects as a result of membrane anchoring and entropic gains upon membrane partitioning caused by the hydrophobic effect. The membrane anchored peptides were not available for interactions with any of the complementary peptides EI or EV, which further indicates membrane partitioning. However, allowing KVC to heterodimerize and fold with EV or EI prior anchoring to the liposome lead to significant and concentration dependent inhibition of the CF release. A subsequent addition of KI or $\mathrm{KV}$ lead to regain in the membrane permeabilizing effect of $\mathrm{KVC}$ due to a heterodimer exchange, which likely was preceded by formation of a membrane bound multimeric peptide complex. In contrast to previous papers on peptide-mediated liposomal release, exploiting either heating $^{12}$ or enzymatic interactions ${ }^{20-22}$ as triggers, the current approach requires relatively low peptide surface concentrations and allows for multiple possibilities for tuning the release rate using 
defined and very specific interactions. The findings presented here thus delineates a new possible strategy for further development of more sophisticated liposomal drug delivery systems.

\section{EXPERIMENTAL SECTION}

General. The lipids 1-palmitoyl-2-oleoyl-sn-glycero-3-phosphocholine (POPC) and 1,2-dioleoylsn-glycero-3-phosphoethanolamine-N-(4-(p-maleimidophenyl)butyramide) (MPB-PE) were acquired from Avanti Polar Lipids (Alabaster, USA). Fmoc-protected amino acids and coupling reagents for Solid-Phase Peptide Synthesis were acquired from Iris Biotech GmbH (Marktredwitz, Germany). Sensor chips L1 and CM5 and all other chemicals and buffers needed for peptide immobilization and regeneration of the sensor surfaces were acquired from GE Healthcare (Uppsala, Sweden). All other chemicals were bought from Sigma Aldrich (Saint Louis, USA).

Peptide synthesis of EI, EV, KI and KV was done in-house using standard Fmoc Solid-Phase Peptide Synthesis protocol as previously described. ${ }^{23}$ In short, the peptides were synthesized at a $0.1 \mathrm{mmol}$ scale on rink amide resin (ChemMatrix, $0.47 \mathrm{mmol}$ of $\mathrm{NH}_{2} /$ resin) using 4 equivalents (equiv) of Fmoc-protected amino acids, 4 equiv of HCTU and 8 equiv of DIPEA. N-terminal acetylation was achieved by adding $10 \mathrm{ml}$ of 1:1 (v/v) acetic anhydride:DMF after the final Fmocdeprotection. The peptides were cleaved from the resin using a $5 \mathrm{ml}$ mixture of TFA:TIPS:MQwater (9.5:0.25:0.25) (v/v) and incubated for $90 \mathrm{~min}$. After filtration to remove the solid support, the resulting TFA/peptide mixtures were concentrated to $1 \mathrm{ml}$ under a flow of $\mathrm{N}_{2}$. The peptides were then precipitated using $-20{ }^{\circ} \mathrm{C}$ diethyl ether and sequentially isolated using centrifugation. The crude peptides were purified using RP-HLPC on a Kromatek HiQ-Sil C18HS column under either acidic- (KI and KV peptides) or alkaline (EI and EV peptides) conditions (peptide purity 
$>95 \%$ ). The final products identity was confirmed using MALDI-ToF mass spectroscopy using a-cyano-hydroxycinnamic acid as a matrix. In addition, peptides with an $\mathrm{N}$-terminal Cys residue (KIC and KVC) were ordered and custom-synthesized from GL Biochem Ltd. and used as received (peptide purity >95\%).

Peptide concentrations were determined by measuring absorbance at $280 \mathrm{~nm}$ (UV-2450 Shimadzu, Japan) by utilizing the Tryptophan present in all sequences.

Liposome preparation was done by thin film hydration and subsequent extrusion. The lipid composition was tailored by mixing MPB-PE an POPC, dissolved in chloroform, in the molar ratios 0:100, 1:99 and 5:95 resulting in 0, 1 and 5 mol\% MPB-PE liposomes. Evaporation of the solvent was done first by a stream of nitrogen and then vacuum desiccation overnight. Liposomes were formed by 10 min rehydration of the dried lipid film with $0.01 \mathrm{M}$ PBS (pH 7.4) followed by 1 min vortexing. To decrease the size polydispersity the liposomes were extruded 21 times through a $100 \mathrm{~nm}$ polycarbonate membrane using a Mini Extruder (Avanti Polar Lipids, USA). For CF encapsulated liposomes, the dry lipid film was instead rehydrated with $50 \mathrm{mM} \mathrm{CF}$ (self-quenching concentration) in $10 \mathrm{mM} \mathrm{PB}$ and $90 \mathrm{mM} \mathrm{NaCl}$ (adjusted to $\mathrm{pH}$ 7.4). The liposome preparation followed as described above with an additional final step of removing the unencapsulated CF by gel filtration using a PD-Mini/Miditrap G-25 column (GE Healthcare, Singapore) eluted with PBS buffer.

Carboxyfluorescein leakage assays were done by using liposomes with CF encapsulated at selfquenching concentration. Leakage was measured as increased fluorescence over time, $\lambda_{\mathrm{ex}}=485$ $\mathrm{nm}$ and $\lambda_{\mathrm{em}}=520 \mathrm{~nm}$ using a fluorescence platereader (BMG Fluostar Galaxy, Germany). The result is presented as percentage of CF released at a chosen timepoint calculated as 
$\%$ CF release $=\frac{\mathrm{F}-\mathrm{F}_{0}}{\mathrm{~F}_{\text {tot }}-\mathrm{F}_{0}} * 100$

where $\mathrm{F}_{0}$ is the initial fluorescence intensity measured before addition of any peptide, $\mathrm{F}$ is the fluorescence intensity at each timepoint and $F_{\text {tot }}$ is the maximum fluorescence corresponding to full release of CF which was achieved by incubation with $1 \%$ Triton X-100. All samples were prepared using PBS buffer (pH 7.4), a total lipid concentration of $40 \mu \mathrm{M}$, a final total volume of $250 \mu \mathrm{L}$ in a 96-well plate and measured in quadruplicates.

Single peptide experiment: Peptide was added to the liposomes at timepoint 0 min. Each final peptide concentration was the result of addition of an equal volume of peptide stocks. The final peptide concentration was $0,0.02,0.05,0.1,0.2,0.5,1,2,5,10,20$ and $50 \mu \mathrm{M}$ and CF release was measured every 2 min for 2 h. Heterodimer experiment: KVC and EV or EI were preincubated 30 min in RT before addition to liposomes, where all additions were of the same volume. Final peptide concentrations were 0,5 and $10 \mu \mathrm{M}$ of KVC and 0, 1, 2, 5, 10, 20 and $50 \mu \mathrm{M}$ EI or EV and CF release was measured every 2 min for 2 h. Heterodimer exchange: $5 \mu \mathrm{M} \mathrm{KVC}$ and $10 \mu \mathrm{M}$ EI (final concentrations) were preincubated $30 \mathrm{~min}$ in RT before addition to liposomes, followed by addition of KI, all KI additions were of the same volume. Final concentrations of KI were 0, 1, 2, 5, 10, 20 and $50 \mu \mathrm{M}$ and CF release was measured every 2 min for $4 \mathrm{~h}$.

Dynamic Light Scattering measurements were done to detect changes in liposome size upon additions of peptides. The size was determined by averaging at least 30 cycles of $10 \mathrm{~s}$ runs with a count rate around $200 \mathrm{kcps}$ and where the result was fitted to the cumulant model using the DLS function of a Zetasizer Nano ZS90 (Malvern, United Kingdom). Liposome and peptide concentrations were chosen to correspond to those of the CF leakage assays. In these experiments 
0, 0.5, 5 and $50 \mu \mathrm{M}$ KIC or KVC was added to $40 \mu \mathrm{M}$ lipid concentration of 0,1 and $5 \mathrm{~mol} \%$ MPB-PE liposomes in PBS (pH 7.4). Samples were measured at 0 and $4 \mathrm{~h}$ in $25^{\circ} \mathrm{C}$.

Circular Dichroism spectroscopy was done with a Chirascan (Applied Photophysics, United Kingdom) using a $1 \mathrm{~mm}$ cuvette and the wavelength recorded was 197-280 nm. Each sample was prepared in PBS buffer (pH 7.4).

Samples with free peptide were prepared at $100 \mu \mathrm{M}$ and incubated in RT for 0, 1, 8, 24, 72 and $168 \mathrm{~h}$. Samples including liposomes were prepared using $50 \mu \mathrm{M}$ peptide and $1 \mathrm{mM}$ total lipids, chosen to achieve an equimolar ratio of thiol:maleimide in the sample with 5 mol\% MPB-PE liposomes.

Surface Plasmon Resonance measurements were run on a Biacore 3000 (GE Healthcare, Sweden) operated at $25^{\circ} \mathrm{C}$. The flowrate was $10 \mu \mathrm{L} / \mathrm{min}$ and PBS were used as the running buffer unless otherwise stated in individual steps.

A L1 sensor chip was used to evaluate peptide-lipid interactions. To minimize unspecific electrostatic binding to the sensor chip the removal of negative charges in the dextran matrix was done by injection of $10 \mathrm{~min} 250 \mu \mathrm{M}$ EDC and $50 \mu \mathrm{M}$ NHS followed by $3.5 \mathrm{~min} 1 \mathrm{mM}$ ethanolamine. Each measurement started with a wash with 2x30 s Isopropanol:50 mM NaOH 2:3 followed by $15 \mathrm{~min}$ injection at $5 \mu \mathrm{L} / \mathrm{min} 0,1$ or $5 \mathrm{~mol} \%$ MPB-PE liposomes at $0.5 \mathrm{mM}$. To remove loosely bound liposomes running buffer was flowed at $100 \mu \mathrm{L} / \mathrm{min}$ for $5 \mathrm{~min}$, followed by a 30 s injection of $10 \mu \mathrm{M} \mathrm{NaOH}$. The peptide injections were always 10 min at $200 \mu \mathrm{M}$ and the post-injection phase was monitored for 10 min. The injection order was always EI/EV (reference injection), then KVC and finally EI/EV. The measurement ended with a 2x30 s Isopropanol:50 $\mathrm{mM} \mathrm{NaOH} \mathrm{2:3} \mathrm{regeneration} \mathrm{step.}$ 
A CM5 sensor chip was used to evaluate peptide-peptide interactions and the heterodimer exchange. The ligands Cys, KIC and KVC were immobilized in different flow channels on the sensor chip using thiol coupling. Immobilization started with wash 30 s 50 mM HCl followed by $10 \mathrm{~min} 250 \mu \mathrm{M}$ EDC and $50 \mu \mathrm{M}$ NHS, $3.5 \mathrm{~min} 18 \mathrm{mg} / \mathrm{mL}$ PDEA in $0.1 \mathrm{M}$ borate buffer pH 8.5, 3.5 min $1 \mathrm{mM}$ ethanolamine, $10 \mathrm{~min} 10 \mu \mathrm{M}$ ligand in acetate buffer $\mathrm{pH}$ 5.5, 3.5 min $100 \mu \mathrm{M}$ Cys and finally 2x30 s $50 \mathrm{mM} \mathrm{HCl}$ to remove any unbound components. Each sample measurement started with a wash of 2x30s $50 \mathrm{mM} \mathrm{NaOH}$ followed by an injection of 10 min $10 \mu \mathrm{M}$ EI, dissociation monitored during $5 \mathrm{~min}$ and a subsequent injection of $10 \mathrm{~min} 0,1,2$ and $5 \mu \mathrm{M} \mathrm{KI}$ or KV. Finally, 2x30s 50 mM NaOH was injected to remove all non-covalently bound peptide.

Isothermal titration calorimetry was done using a Microcal PEAQ-ITC (Malvern, United Kingdom). The experiments were performed by titrating $30 \mathrm{mM} 0$ or 5 mol\% MPB-PE liposomes into $150 \mu \mathrm{M}$ Cys or KVC. Each injection was $3 \mu \mathrm{L}$ and the equilibration time between titrations was set to $5 \mathrm{~min}$. The peaks corresponding to the injections were then integrated with respect to a fitted baseline in order to get the $\mathrm{kcal} / \mathrm{mol}$ injectant.

\section{ASSOCIATED CONTENT}

Supporting Information. Peptide sequences and helical wheel diagrams; lipids and liposome composition; CF release kinetics for KIC and KVC; CF release profiles without peptides and for peptides without Cys; CF release with partly oxidized KIC and KVC; peptide oxidation rates and folding of KIC and KVC; size distribution of 5 mol\% MPB-PE liposomes with KVC; additional SPR data on heterodimerization (EVKVC) on KVC-functionalized liposomes; ITC data on 
interaction of KVC with 0 and 5 mol\% MPB-PE liposomes; calculations of free KVC as a function of EI/EV; SPR data on the heterodimer exchange of EVCKI with EI/EV

\section{AUTHOR INFORMATION}

\section{Corresponding Author}

*E-mail: daniel.aili@liu.se

\section{Author Contributions}

The manuscript was written through contributions of all authors. All authors have given approval to the final version of the manuscript.

\section{ACKNOWLEDGMENT}

The authors kindly acknowledge the finical support from the Swedish Research Council (VR), grant number 2017-04475, and the Swedish Cancer Foundation, grant number CAN 2017/430, the Swedish Government Strategic Research Area in Materials Science on Functional Materials at Linköping University (Faculty Grant SFO-Mat-LiU No. 2009-00971). During this study CS was enrolled in the graduate school Forum Scientium.

\section{REFERENCES}

(1) Guidotti, G.; Brambilla, L.; Rossi, D. Cell-Penetrating Peptides: From Basic Research to Clinics Trends Pharmacol. Sci. 2017, 38 (4), 406-424. 
(2) Hancock, R. E. W.; Chapple, D. S. Peptide Antibiotics Antimicrob. Agents Chemother. 1999, 43 (6), 1317-23.

(3) Brogden, K. A. Antimicrobial Peptides: Pore Formers or Metabolic Inhibitors in Bacteria? Nat. Rev. Microbiol. 2005, 3 (3), 238-250.

(4) Zasloff, M. Antimicrobial Peptides of Multicellular Organisms Nature 2002, 415 (6870), 389-395.

(5) Rothbard, J. B.; Garlington, S.; Lin, Q.; Kirschberg, T.; Kreider, E.; McGrane, P. L.; Wender, P. A.; Khavari, P. A. Conjugation of Arginine Oligomers to Cyclosporin A Facilitates Topical Delivery and Inhibition of Inflammation Nat. Med. 2000, 6 (11), $1253-1257$.

(6) Oehlke, J.; Scheller, A.; Wiesner, B.; Krause, E.; Beyermann, M.; Klauschenz, E.; Melzig, M.; Bienert, M. Cellular Uptake of an $\alpha$-Helical Amphipathic Model Peptide with the Potential to Deliver Polar Compounds into the Cell Interior Non-endocytically BBA - Biomembranes. 1998, 1414 (1-2), 127-139.

(7) Gomes, B.; Augusto, M. T.; Felício, M. R.; Hollmann, A.; Franco, O. L.; Gonçalves, S.; Santos, N. C. Designing Improved Active Peptides for Therapeutic Approaches against Infectious Diseases Biotechnol. Adv. 2018, In press (doi.org/10.1016/j.biotechadv.2018.01.004).

(8) Fjell, C. D.; Hiss, J. A.; Hancock, R. E. W.; Schneider, G. Designing Antimicrobial Peptides: Form Follows Function Nat. Rev. Drug Discov. 2012, 11 (1), 37-51.

(9) Versluis, F.; Voskuhl, J.; Van Kolck, B.; Zope, H.; Bremmer, M.; Albregtse, T.; Kros, A. In Situ Modification of Plain Liposomes with Lipidated Coiled Coil Forming Peptides Induces Membrane Fusion J. Am. Chem. Soc. 2013, 135 (21), 8057-8062.

(10) Robson Marsden, H.; Korobko, A. V.; Zheng, T.; Voskuhl, J.; Kros, A. Controlled Liposome Fusion Mediated by SNARE Protein Mimics Biomater. Sci. 2013, 1 (10), 1046-1054. 
(11) Futaki, S.; Ohashi, W.; Suzuki, T.; Niwa, M.; Tanaka, S.; Ueda, K.; Harashima, H.; Sugiura, Y. Stearylated Arginine-rich Peptides: A New Class of Transfection Systems Bioconjugate Chem. 2001, 12 (6), 1005-1011.

(12) Al-Ahmady, Z. S.; Al-Jamal, W. T.; Bossche, J. V.; Bui, T. T.; Drake, A. F.; Mason, A. J.; Kostarelos, K. Lipid-peptide Vesicle Nanoscale Hybrids for Triggered Drug Release by Mild Hyperthermia in Vitro and in Vivo ACS Nano 2012, 6 (10), 9335-9346.

(13) Lim, S. K.; Sandén, C.; Selegård, R.; Liedberg, B.; Aili, D. Tuning Liposome Membrane Permeability by Competitive Peptide Dimerization and Partitioning-Folding Interactions Regulated by Proteolytic Activity Sci. Rep. 2016, 6, 21123.

(14) Banerjee, J.; Hanson, A. J.; Gadam, B.; Elegbede, A. I.; Tobwala, S.; Ganguly, B.; Wagh, A. V.; Muhonen, W. W.; Law, B.; Shabb, J. B.; Srivastava, D. K.; Mallik, S. Release of Liposomal Contents by Cell-Secreted Matrix Metalloproteinase-9 Bioconjugate Chem. 2009, 20 (7), 1332-1339.

(15) Bozzuto, G.; Molinari, A. Liposomes as Nanomedical Devices Int. J. Nanomed. 2015, 10, 975-999.

(16) Allen, T. M.; Chonn, A. Large Unilamellar Liposomes with Low Uptake into the Reticuloendothelial System FEBS Lett. 1987, 223 (1), 42-46.

(17) Gabizon, A.; Papahadjopoulos, D. Liposome Formulations with Prolonged Circulation Time in Blood and Enhanced Uptake by Tumors Proc. Natl. Acad. Sci. U.S.A. 1988, 85, 6949-6953.

(18) Allen, T. M.; Hansen, C.; Martin, F.; Redemann, C.; Yan-Young, A. Liposomes Containing Synthetic Lipid Derivates of Poly(ethylene glycol) show Prolonged Circulation Half-lives in Vivo BBA - Biomembranes. 1991, 1066 (1), 29-36.

(19) Barenholz, Y. Doxil ${ }^{\circledR}$ - The First FDA-approved Nano-drug: Lessons learned $J$. Controlled Release 2012, 160 (2), 117-134. 
(20) Terada, T.; Iwai, M.; Kawakami, S.; Yamashita, F.; Hashida, M. Novel PEG-matrix Metalloproteinase-2 Cleavable Peptide-lipid Containing Galactosylated Liposomes for Hepatocellular Carcinoma-selective Targeting J. Controlled Release 2006, 111 (3), 333342.

(21) Wan, Y.; Han, J.; Fan, G.; Zhang, Z.; Gong, T.; Sun, X. Enzyme-responsive Liposomes Modified Adenoviral Vectors for Enhanced Tumor Cell Transduction and Reduced Immunogenicity Biomaterials 2013, 34 (12), 3020-3030.

(22) Kulkarni, P. S.; Haldar, M. K.; Nahire, R. R.; Katti, P.; Ambre, A. H.; Muhonen, W. W.; Shabb, J. B.; Padi, S. K. R.; Singh, R. K.; Borowicz, P. P.; Shrivastava, D. K.; Katti, K. S.; Reindl, K.; Guo, B.; Mallik, S. MMP-9 Responsive PEG Cleavable Nanovesicles for Efficient Delivery of Chemotherapeutics to Pancreatic Cancer Mol. Pharm. 2014, 11 (7), 2390-2399.

(23) Aronsson, C.; Dånmark, S.; Zhou, F.; Öberg, P.; Enander, K.; Su, H.; Aili, D. Self-sorting Heterodimeric Coiled Coil Peptides with Defined and Tuneable Self-assembly Properties Sci. Rep. 2015, 5, 14063.

(24) Dånmark, S.; Aronsson, C.; Aili, D. Tailoring Supramolecular Peptide-Poly(ethylene glycol) Hydrogels by Coiled Coil Self-Assembly and Self-Sorting Biomacromolecules 2016, 17 (6), 2260-2267.

(25) Thomas, F.; Boyle, A. L.; Burton, A. J.; Woolfson, D. N. A Set of de Novo Designed Parallel Heterodimeric Coiled Coils with Quantified Dissociation Constants in the Micromolar to Sub-nanomolar Regime J. Am. Chem. Soc. 2013, 135 (13), 5161-5166.

(26) Litowski, J. R.; Hodges, R. S. Designing Heterodimeric Two-stranded $\alpha$-Helical CoiledCoils: Effects of Hydrophobicity and $\alpha$-Helical Propensity on Protein Folding, Stability, and Specificity J. Biol. Chem. 2002, 277 (40), 37272-37279.

(27) Zhu, B.-Y.; Zhou, N. E.; Kay, C. M.; Hodges, R. S. Packing and Hydrophobicity Effects on Protein Folding and Stability: Effects of Beta-branched Amino Acids, Valine and 
Isoleucine, on the Formation and Stability of Two-Stranded $\alpha$-Helical Coiled Coils/Leucine Zippers Protein Sci. 1993, 2 (3), 383-394.

(28) Ellman, G. L. Tissue sulfhydryl groups Arch. Biochem. Biophys. 1959, 82 (1), 70-77.

(29) Kyte, J.; Doolittle, R. F. A Simple Method for Displaying the Hydropathic Character of a Protein J. Mol. Biol. 1982, 157 (1), 105-132.

(30) Wimley, W. C.; White, S. H. Experimentally Determined Hydrophobicity Scale for Proteins at Membrane Interfaces Nat. Struct. Biol. 1996, 3 (10), 842-848.

(31) Lau, S. Y.; Taneja, A. K.; Hodges, R. S. Synthesis of a Model Protein of Defined Secondary and Quaternary Structure: Effect of Chain Length on the Stabilization and Formation of Two-stranded $\alpha$-Helical Coiled-Coils J. Biol. Chem. 1984, 259 (21), $13253-13261$.

(32) Kaiser, E. T.; Kézdy, F. J. Secondary Structures of Proteins and Peptides in Amphiphilic Environments (a Review) Proc. Natl. Acad. Sci. U.S.A. 1983, 80 (4), 1137-1143.

(33) Bechinger, B.; Lohner, K. Detergent-like Actions of Linear Amphipathic Cationic Antimicrobial Peptides BBA - Biomembranes. 2006, 1758 (9), 1529-1539.

(34) Liu, J.; Conboy, J. C. Direct Measurement of the Transbilayer Movement of Phospholipids by Sum-Frequency Vibrational Spectroscopy J. Am. Chem. Soc. 2004, 126 (27), 8376-8377.

(35) Rabe, M.; Zope, H. R.; Kros, A. Interplay between Lipid Interaction and Homo-coiling of Membrane-Tethered Coiled-Coil Peptides Langmuir 2015, 31 (36), 9953-9964. 
TABLE OF CONTENTS GRAPHICS (For Table of Contents Only)

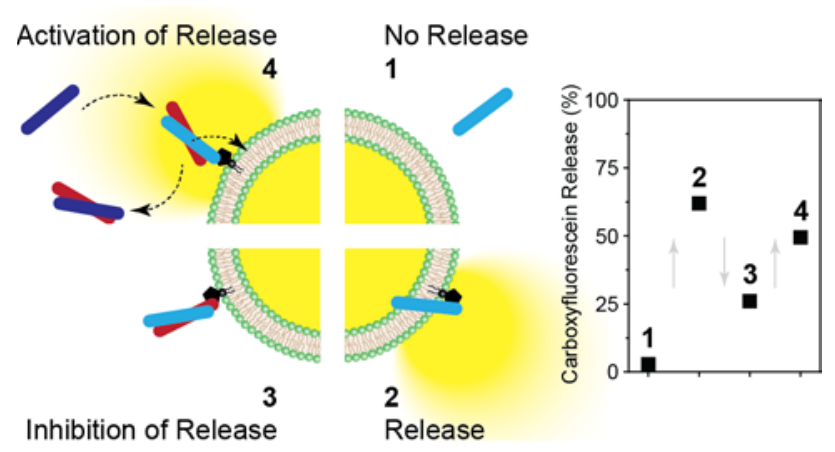

